\title{
Lokale Bündnisse für Familie - Ein Handlungsfeld für Gewerkschaften
}

\section{„ALLIANZ FÜR DIE FAMILIE“}

Familie und Beruf im Alltag unter einen Hut $\mathrm{zu}$ bringen, ist hierzulande immer noch schwer. Initiativen für eine bessere Balance von Familie und Arbeitswelt bündelt die „Allianz für die Familie“. Partner sind die Spitzenverbände der deutschen Wirtschaft, Gewerkschaften (DGB und IG BCE), die Bertelsmann- und die HertieStiftung, renommierte Unternehmen (z. B. BASF, Deutsche Bahn) sowie namhafte Ökonomen und Persönlichkeiten aus der Politik. Sie haben sich im Oktober 2006 mit Bundeskanzlerin Angela Merkel und Bundesfamilienministerin Ursula von der Leyen auf gemeinsame Grundsätze und neue Wege zu einer familienbewussten Arbeitswelt verständigt.

Neben vielen Handlungsfeldern im Betrieb wurde die lokale Ebene als wichtig erkannt. Zugleich wurde in einer gemeinsamen Erklärung der Partner die Unterstützung der Bundesinitiative „Lokale Bündnisse für Familie" betont. Die meisten Allianzpartner aus Politik und Wirtschaft engagieren sich deshalb wie der DGB-Vorsitzende Michael Sommer im Kuratorium als Botschafter der Initiative. Denn eine nachhaltige Familienpolitik muss auf betrieblicher und auch auf lokaler Ebene umgesetzt werden, da eine bessere Vereinbarkeit von Beruf und Familie nicht allein im Unternehmen zu erreichen ist. Sie erfordert vielmehr auch überbetriebliche Lösungsstrategien. Zweierlei wird gebraucht: Arbeitsbedingungen, die Vätern und Müttern eine gute Balance von Beruf und Familie ermöglichen, und attraktive Lebensbedingungen für Familien vor Ort inklusive hochwertiger, flexibler Kinderbetreuungsangebote. Das sind langjährige, gewerkschaftliche Forderungen und zugleich zentrale Handlungsfelder der Lokalen Bündnisse für Familie. Oft lassen sich deshalb konkrete betriebliche Initiativen zur Verbesserung der Kinderbetreuungssituation der Beschäftigten effektiv mit den gewerkschaftlichen Aktivitäten in den Lokalen Bündnissen koppeln bzw. sie verstärken sich durch Synergieeffekte. Gute Beispiele sind die lokalen Bündnisse für Familie in Trier, Hanau, auf Rügen und in Jena.

Für die Gewerkschaften bieten sich in Lokalen Bündnissen Handlungsfelder auf kommunaler Ebene, die weit über das betriebliche Engagement hinausgehen: soziale Integration und Vermeidung von Ausgrenzung oder gar des sozialen Abdriftens ganzer Stadtquartiere, eine Verbesserung der Beschäftigungschancen von Müttern und der Bildungschancen von Kindern.

\section{AKTEURSNETZWERKE BÜNDELN RESSOURCEN UND NUTZEN SYNERGIEEFFEKTE}

Über 430 Bündnisse haben sich bisher der 2004 von der damaligen Bundesfamilienministerin Renate Schmidt gestarteten Initiative „Lokale Bündnisse für Familie“ angeschlossen. Die jeweiligen Bündnispartner aus Politik, Verwaltung, Wirtschaft sowie Gewerkschaften, freie Träger, soziale Einrichtungen, Kirchengemeinden und Initiativen arbeiten vereint daran, Rahmenbedingungen für Familien konkret zu gestalten.

Die Bündnisse können Kontaktplattform, Diskussionsforum, Ideenschmiede und Lobby für Familien sein. Sie leben vom Engagement ihrer Akteure, orientieren sich an den konkreten Bedürfnissen von Familien und setzen auf passgenaue regionale Lösungsansätze: für eine bessere Vereinbarkeit von Familie und Beruf, für eine flexible Kinderbetreuung, für eine gute und frühe Förderung von Kindern, für maßgeschneiderte Dienstleistungen für Familien, für Unterstützung von Eltern und für ein familienfreundliches Umfeld. Mehr als 3000 Einzelprojekte werden über die große Bandbreite an Themen und Aufgaben hinweg in den Bündnissen umgesetzt.

So erhalten z. B. im Landkreis Leer berufstätige Mütter Rat und Unterstützung zu Fragen der beruflichen Weiterbildung und bei der Suche nach Arbeitsplätzen. In Bocholt und Berlin werden "Leih-Omas" vermittelt. In Rietberg bietet ein Notfall-
mutter-Projekt Hilfe an, in Solingen und Recklinghausen werden Tagesmütter ausgebildet und vermittelt, in Gütersloh Eltern geschult, mit Stresssituationen umzugehen. All diese Maßnahmen entlasten berufstätige Eltern. Im Chemnitzer Bündnis wurde unter Federführung der DGB-Region ein Generationentreff eingerichtet, um Jung und Alt im Stadtteil zusammenzubringen.

Diese und viele andere konkrete, innovative Aktivitäten haben Lokale Bündnisse für Familie entwickelt, um Städte, Gemeinden und Kreise gezielt familienfreundlicher zu gestalten. Die Bündnisprojekte werden stets durch eine Vielzahl von Akteuren und deren unterschiedliches, sich ergänzendes Engagement ermöglicht: Die einen stiften Geld, die anderen Know-how, die Dritten ehrenamtliches Personal und die Vierten Räumlichkeiten.

Das läuft bei der Vielfalt der Akteure nicht immer einvernehmlich und konfliktfrei ab. Doch solche Netzwerke funktionieren nur, wenn die Akteure bereit sind, Ressourcen zu bündeln und im Sinne der gemeinsamen Zielsetzung auch einzusetzen. Nur dann kann die Bündnisarbeit die verschiedenen Kompetenzen zusammenführen, Ressourcen sparen und Raum für neue Ideen schaffen.

Das Spektrum Lokaler Bündnisse für Familie bietet eine große Vielfalt von Lösungsansätzen. Sie ermöglichen zum einen kurzfristige Maßnahmen, um die Situation von Familien zu verbessern, zum anderen können nachhaltige Strategieprozesse mit langfristiger Standort-Perspektive entwickelt werden. Ökonomische, sozialwissenschaftliche und publizistische Untersuchungen bestätigen, dass sich Familienfreundlichkeit für alle Beteiligten lohnt und auch dank der Lokalen Bündnisse zum be-

Christina Stockfisch, Dr., Leiterin des Projektes "Vereinbarkeit von Familie und Beruf gestalten!" beim DGB-Bundesvorstand. e-mail: christina.stockfisch@dgb.de 
deutsamen Standortfaktor für Kommunen, Regionen und Unternehmen wird. Für die Kommunen ist das Engagement im Bündnis für eine bessere Balance ein wichtiger Standortvorteil. Die demografische Entwicklung zwingt Städte und Gemeinden in einen immer härteren Wettbewerb. Bündnisse für Familienfreundlichkeit steigern die Attraktivität gerade für junge $\mathrm{Fa}$ milien und stärken dadurch die regionale Wirtschaftskraft.

Unternehmen profitieren durch die Zusammenarbeit in der Umsetzung familienorientierter Leistungen, z. B. durch Belegplätze in Kindertageseinrichtungen oder durch Weiterbildungsangebote für Beschäftigte in Elternzeit. Im Bündnis können gerade kleine und mittelständische Unternehmen familienfreundliche Maßnahmen umsetzen, die für ein einzelnes Unternehmen zu aufwendig wären. Unternehmen sind in drei Vierteln der Bündnisse vertreten. Laut einer Prognos-Studie zahlen sich Firmeninvestitionen in Familienfreundlichkeit mit einer Rendite von bis zu $25 \%$ aus, weil sie Kosten für Neueinstellungen, Überbrückungs- und Fehlzeiten senken, Fachkräfte motivieren und ans Unternehmen binden.

Auch für Gewerkschafterinnen und Gewerkschafter birgt ein verstärktes Engagement in diesem Bereich große Chancen und eröffnet eine weitere Zukunftsperspektive. Work-Life-Balance als Gegenstand gewerkschaftlicher Politik erfordert keinen Bruch mit den Traditionen, sondern deren Weiterentwicklung im Licht neuer Realitäten. Gewerkschaften können sich hier gezielt überbetrieblich für die Interessen der Beschäftigten engagieren und als treibende Kräfte eines positiven Kulturwandels auftreten, bei dem wirtschaftliche Dynamik und soziale Rücksicht keine Gegensätze sind. Damit werden sie auch attraktiver für solche Zielgruppen, die bei ihnen bisher eher unterrepräsentiert sind: Frauen, Jüngere, Beschäftigte in den neuen Berufen.

Zurzeit engagieren sich Gewerkschafter und Betriebsräte in knapp 100 Bündnissen für Familie und bringen ihr Wissen um die Bedürfnisse der Beschäftigten bezüglich Arbeitszeitflexibilisierung und Kinderbetreuung ein. Damit vertreten sie die Beschäftigteninteressen in ca. einem Viertel der gegründeten Bündnisse; die anderen drei Viertel arbeiten noch ohne Gewerkschaftsbeteiligung. Impulse zur Vernetzung und Ausweitung gewerkschaftlicher Akti- vitäten in lokalen Bündnissen gab das DGB-Projekt „Vorfahrt für Familien!“, einer von vier Innovationspiloten der Bundesinitiative, deren Ergebnisse in der Publikation "Von Bündnissen für Bündnisse" dargestellt werden. ${ }^{1}$

\section{DGB-PROJEKT „VORFAHRT FÜR FAMILIEN! " : INNOVATIONSPILOTEN GEBEN IMPULSE}

In Hanau und Jena, auf der Insel Rügen und beim Deutschen Gewerkschaftsbund wurden mit Mitteln des Bundesministeriums für Familie, Senioren, Frauen und Jugend (BMFSFJ) und des Europäischen Sozialfonds über einen Zeitraum von zwei Jahren (2005/2006) Ansätze und Lösungen für die Vereinbarkeit von Familie und Beruf entwickelt und umgesetzt.

Das DGB-Projekt „Vorfahrt für Familien!" vernetzte gewerkschaftliche und betriebliche Aktivitäten regional und bundesweit. Betriebsräte und gewerkschaftlich Engagierte wurden für die Vereinbarkeit von Familie und Beruf sensibilisiert und qualifiziert. Ein umfangreicher Werkzeugkoffer bietet allen Interessierten nunmehr gebündelt Anregungen und Erfahrungen zu gewerkschaftlichen Bündnisaktivitäten sowie Konzepte und umfassende Materialien zu zentralen Handlungsfeldern der Vereinbarkeit von Familie und Beruf, z. B. $\mathrm{zu}$ den Themen Elternzeit und Wiedereinstieg, familienfreundliche Arbeitszeitmodelle, betrieblich geförderte Kinderbetreuung. ${ }^{2}$

Zeit steht im Mittelpunkt der Hanauer Bündnisaktivitäten. Der Innovationspilot hat z. B. durch familienfreundliche "Zeitbrücken“, ,Zeitfenster“ und „Zeitinseln“eine Flexibilisierung von Öffnungs- und Servicezeiten von Handwerksbetrieben und Arztpraxen geschaffen und so zur Entlastung von berufstätigen Eltern beigetragen.

Der „Familienservice im Familienzentrum Jena" ist Anlauf- und Koordinationsstelle für Familien und Unternehmen bei Fragen zu flexibler Kinderbetreuung. Neben der Vermittlung von Kinderfrauen und Babysittern betreibt er einen Mini-Kindergarten „Zwergenkiste“ für Kinder unter drei Jahren. Die Zwergenkiste soll Familien, bei denen ein Elternteil in der Elternzeit stundenweise arbeiten geht, selbstständig tätigen Frauen und Alleinerziehenden, die den Wiedereinstieg in den Beruf finden wollen, eine Entlastungsmöglichkeit bieten.
Beim Rügener Bündnis stehen besondere Herausforderungen und Lösungen für flexible Kinderbetreuungsangebote einer touristisch geprägten Region im Mittelpunkt. Die FlexiKIDS bieten Randzeitenbetreuung für Kinder, die sowohl von Beschäftigten auf der Insel als auch von Familien im Urlaub genutzt werden kann.

\section{FAMILIENFREUNDLICHKEIT VOR ORT GESTALTEN: CHANCEN UND RISIKEN LOKALER BÜNDNISSE}

Der Siebte Familienbericht der Bundesregierung sieht in den Lokalen Bündnissen einen wichtigen Baustein nachhaltiger Familienpolitik, denn familienfreundliche Bedingungen werden in unserer Gesellschaft verstärkt auch dezentral geschaffen, um schnell und wirksam zu konkreten Lösungen vor Ort zu gelangen. Damit sind Chancen, aber auch Risken verbunden. Wir sehen in den Lokalen Bündnissen die Chance, dass sich eine Form von Politik entwickelt, die sich tatsächlich an den Bedürfnissen der Menschen orientiert, weil sie durch ihre Organisationsform als „runder Tisch" vom Prinzip her niemand ausschließt und die Menschen selbst für ihre konkreten Anliegen aktiv werden können. Wichtig ist allerdings, dass bisherige Aktivitäten im Politikfeld eingebunden werden (z. B. die Jugendhilfeausschüsse) und sich keine informellen Parallelstrukturen entwickeln.

Auch die Rolle des bürgerschaftlichen Engagements in den Prozessen der Lokalen Bündnisse sollte man genauer analysieren. Formell sind alle aufgefordert, sich an den Bündnissen zu beteiligen. Doch wer sich beteiligt und was dabei herauskommt, liegt in der Verantwortung der lokal Handelnden. Das sind in den Bündnissen für Familie meist Vertreter der Kommune, der Wirtschaftsverbände und einzelner Unternehmen. So stellt sich die Frage, ob z. B. sozial schwache Familien und Familien mit Migrationshintergrund überhaupt angesprochen und in die Bündnisarbeit vor Ort einbezogen werden. Auch Kinder, deren Eltern nicht in Erwerbsstrukturen eingebunden sind, benötigen dringend qualifizierte Angebote.

\footnotetext{
1 Download unter www.bmfsfj.de und www.lokalebuendnisse-fuer-familie.de

2 Download unter www.familie.dgb.de/projekt/ tools
} 
Solange sich aber nur Teilgruppen, wie z. B. bildungsorientierte, berufstätige sowie bürgerliche Schichten engagieren, können auch nur isolierte Inseln in einem bestimmten Quartier, an einer Schule, innerhalb eines begrenzten Netzwerkes oder Ähnliches geschaffen werden. Doch solche Inseln allein reichen nicht aus, um die Rahmenbedingungen für Familien vor Ort nachhaltig zu verbessern.

In Lokalen Bündnissen sind pragmatisches Handeln und schnell vorzeigbare Ergebnisse gefragt. Doch die Beteiligung an den Lokalen Bündnissen verpflichtet nicht zwangsläufig auch zu Qualitätskriterien, die Anforderungen an Partizipation, Chancengleichheit und inhaltliche Qualität beim Prozessmanagement und den Ergebnissen genügen. Die Gewerkschaften achten als Akteure in diesen Netzwerken deshalb darauf, dass nicht nur gute Ergebnisse erreicht werden, sondern auch, dass diese gemeinwohlorientiert sind. Damit sich lokale Familienbündnisse nicht zu aktivistischen Strohfeuern, sondern zu erfolgreichen Netzwerken entwickeln, müssen Qualität und Nachhaltigkeit gesichert werden, z. B. bezüglich der eigenen Strukturen, der Kinderbetreuungsangebote und der Frauenerwerbstätigkeit.

Darüber hinaus bringen die Gewerkschaften ihr spezielles Profil in die Lokalen Bündnisse ein: Als Interessenvertretung der Beschäftigten in den Unternehmen einerseits und in den Bereichen Bildung und Erziehung andererseits engagieren sie sich vor allem für Chancengleichheit von Frauen und Männern sowie von Kindern unterschiedlicher sozialer und ethnischer Herkunft.

\section{NACHMACHEN ERWÜNSCHT}

Das Rad wird in den einzelnen Bündnissen nicht immer wieder neu erfunden - die Bündnisse vernetzen sich und lernen voneinander. Denn die Kernthemen ähneln sich oft: der Wunsch berufstätiger Eltern nach flexibler Kinderbetreuung, nach einem familiengerechten Wohnumfeld und effektiven Zeitmanagement, die Förderung generationenübergreifender Zusammenarbeit.

Die Verbesserung der Kinderbetreuungssituation, z. B. durch die Einrichtung betrieblicher und überbetrieblicher Kindertagesstätten, ist dabei in westdeutschen Regionen oft der dringendste Schwerpunkt. In Ostdeutschland geht es vielfach darum, die Abwanderung junger Menschen vor oder während der Familiengründungsphase zu stoppen.

In jedem Falle hilft der Austausch mit anderen Bündnisaktiven über manche Hürde und unterstützt mit praktischen, übertragbaren Ideen und Lösungen. Das Servicebüro der Bundesinitiative unterstützt solche Vernetzungsprozesse und organisiert den Wissens- und Erfahrungsaustausch der Einzelbündnisse durch regelmäßige landesweite Netzwerktreffen sowie thematische Fach- und Diskussionsforen.

\section{HERAUSFORDERUNGEN FÜR DIE DYNAMISCHE INITIATIVE}

Die Dynamik, mit der die Bündnisidee in den letzten drei Jahren aufgegriffen und örtlich umgesetzt wurde, verdeutlicht zweierlei: Erstens muss unsere Gesellschaft die Situation von Familien effektiv und nachhal- tig verbessern, um sozial, demografisch und wirtschaftlich zukunftsfähig zu bleiben bzw. zu werden.

Zweitens scheint der Bündnisansatz den aktuellen gesellschaftlichen Gestaltungsdrang gut aufzufangen. Der renommierte Publizist Warnfried Dettling lobte die Bündnisse als gesellschaftliche Impulsgeber, die als „soziale Intelligenzverstärker“ Lebensqualität schaffen, örtliche Vielfalt gestalten und Synergien freisetzen. „Die lokalen Bündnisse für die Familien gehören zu den erfolgreichen Beispielen in Deutschland, denen es gelungen ist, die wichtigen Akteure der lokalen Gesellschaft an einen Runden Tisch zu bringen, an dem sie auf gemeinsame Ziele hin kooperieren". 3

Die Bündnisse geben also neuen Schub, Deutschland in vielen kleinen Schritten kinder- und familienfreundlicher zu gestalten. Das ist in unserem kinderentwöhnten Land dringend nötig.

Die große Herausforderung ist es nunmehr, die Qualität und Nachhaltigkeit der vielfältigen lokalen Ansätze zu sichern und so die derzeitige Aufbruchstimmung in eine wirksame und kontinuierliche Bewegung zu überführen.

\footnotetext{
3 Warnfried Dettling (2005): Neue Dynamik in der Familienpolitik. Lokale Bündnisse als gesellschaftliche Impulsgeber.
} 